\title{
A GAME THEORETIC FRAMEWORK FOR INCENTIVE-BASED PEER-TO-PEER LIVE-STREAMING SOCIAL NETWORKS
}

\author{
W. Sabrina Lin*, H. Vicky Zhao ${ }^{\dagger}$ and K. J. Ray Liu* \\ * ECE Dept., University of Maryland, College Park, MD 20742 USA \\ ${ }^{\dagger}$ ECE Dept., University of Alberta, Edmonton, AB T6G 2V4 Canada
}

\begin{abstract}
Multimedia social network analysis is an emerging research area, which analyzes the behavior of users who share multimedia content and investigates the impact of human dynamics on multimedia systems. In peer-to-peer live-streaming social networks, user cooperate with each other to provide a distributed, highly scalable and robust platform for live streaming applications. However, every user wishes to use as much bandwidth as possible to receive a high-quality video, and full cooperation cannot be guaranteed. This paper proposes a game-theoretic framework to model user behavior and designs incentive-based strategies to stimulate user cooperation in peer-to-peer live streaming. We analyze the Nash equilibrium and the Pareto optimality of the game. We also take into consideration selfish users' cheating behavior and propose cheat-proof strategies. Both our analytical and simulation results show that the proposed strategies can effectively stimulate user cooperation, achieve cheat free and help provide reliable services.
\end{abstract}

Index Terms - Multimedia social network, user dynamics

\section{INTRODUCTION}

With recent advance in networking, multimedia processing, and communications, over millions of users share multimedia data over Internet, and we witness the emergence of largescale multimedia social networks. In such large scale social networks, users influence each other's decisions and performance, and it raises a critical issue to formulate the complex user dynamics and analyze the impact of human factors on multimedia systems. Such investigation provides fundamental guidelines to offer secure and personalized services.

Peer-to-Peer (P2P) live streaming [1], is one of the biggest multimedia social networks on the internet, consisting of selforganized and distributed systems without centralized authorities or infrastructures. Users in a P2P live-streaming social network watch live programs over networks simultaneously, and the system relies on voluntary contributions of resources

\footnotetext{
The authors can be reached at wylin@eng.umd.edu, vzhao@ece.ualberta.ca, and kjrliu@eng.umd.edu.
}

from individual users to achieve high scalability and robustness. Each user has limited bandwidth and wants to maximize his/her own payoff. Different users have different objectives and full user cooperation cannot be guaranteed. Therefore, to provide satisfactory services, a critical issue to be resolved first is to analyze users' behavior, provide incentives and design optimal strategies to stimulate user cooperation [2].

Users in a P2P live streaming social network are strategic and rational, and they are likely to manipulate any incentive systems to maximize their own payoff. Every rational user in the network is selfish and wants to receive the highest possible resolution of the video. They will even cheat if they believe it could help maximize their payoff. Game theory [3] is a proper tool to model the interaction among peers, and to analyze the optimal and cheat-proof cooperation strategies.

In the literature, a game theoretic framework was proposed in [4] for P2P file sharing and [5], [6] model and give incentives in P2P file sharing. The work in [7] introduced an incentive-based cooperation mechanism for P2P live streaming networks, assuming every user is willing to cooperate. In [8], a reputation-based mechanism was proposed to stimulate user cooperation in P2P live-streaming networks. However, all prior work do not consider the cheating behavior of selfish users and do not address the cheat-proof issues in P2P livestreaming social networks.

In this paper, we focus on designing cooperation stimulation strategies for P2P live streaming social networks under a game theoretic framework. We first study a two-player game and investigate the Nash equilibrium. Since this game usually has multiple equilibriums, we then investigate how to apply extra optimality criteria, such as Pareto optimality, fairness, and cheat-proofing, to further refine the obtained Nash equilibrium solutions. The goal of this analysis is to stimulate each pair of user in the P2P live streaming game to cooperate with each other and achieve better performance.

The rest of this paper is organized as follows. Section 2 introduces the P2P live streaming system model. Section 3 studies the two-player game and the equilibria. In Section 4 we show simulation results to evaluate the performance of the proposed strategies. Finally, Section 5 concludes this paper. 


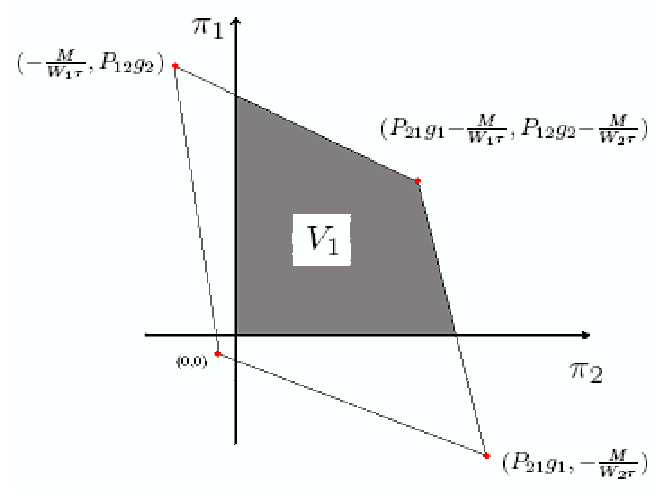

Fig. 1. Feasible and Enforceable payoff profiles

\section{SYSTEM MODEL}

\subsection{P2P Live Streaming Cooperation Model}

In a delivery architecture for live video streaming, a video bit stream is divided into media chunks of $M$ bits, and all the chunks are available at an original server. When a peer wants to view the video, he/she first obtains a list of peers currently watching the video, together with information about the availability of each chunk in others' buffers. At the beginning of each round, every user sends a request either to one of his peers or the original server. Every peer can only send one request in each round and also answer at most one request. Let $\tau$ be the duration of each round.

\subsection{Two-Player Game Model}

To simplify the analysis, we start from the two-person game with single-layer video coding structure. There are total $N$ users in the social network, and the original server's upload bandwidth can only afford transmitting $N^{\prime}$ chunks in $\tau$ seconds, and $N^{\prime}<<N$. Assume at the beginning, every user in the network only asks the original server for the data, and two of them, user 1 and user 2, want to see if they can cooperate with each other for to get a better-quality video.

In the two person game, if player $i$ answers the other player $k$ 's request and sends the requested chunk of data to $\mathrm{him} / \mathrm{her}$, then player $i$ 's cost is $M /\left(W_{i} \tau\right)$, where $W_{i}$ is player $i$ 's available upload bandwidth. On the other hand, if player $k$ also forwards the data that $i$ requested to him/her and player $i$ receives the chunk correctly, then he gets a gain of $g_{i}$. Here, the cost is considered as the percentage of upload bandwidth occupied by transmitting the chunk and the gain is an user defined value between 0 and 1 . It is reasonable to assume that $g_{i} \geq c_{i}$ and there exists a $c_{\max }$ with $c_{i} \leq c_{\max }$, which is the same as if there exists a minimum upload bandwidth $W_{\text {min }}$ such that $W_{i} \geq W_{\min }$. Here, $W_{i}$ and $g_{i}$ are player $i$ s private information, which is not known to the other player unless player $i$ reports them either honestly or dishonestly.

In each round, player $i$ can choose its action $a_{i}$ from 0,1 , where $a_{i}=0$ means in this round, player $i$ chooses not to re- spond to the other player's request, while $a_{i}=1$ means player $i$ is willing to cooperate at this round. $P_{12}$ and $P_{21}$ denote the successful transmission probability from user 1 to user 2 and that from user 2 to user 1, respectively. Then, for each round, players' payoffs are calculated as follows, provided that the action profile $\left(a_{1}, a_{2}\right)$ being taken:

$$
\begin{aligned}
& \pi_{1}\left(a_{1}, a_{2}\right)=\left(a_{2} P_{21}\right) g_{1}-a_{1} \frac{M}{W_{1} \tau} \\
& \pi_{2}\left(a_{1}, a_{2}\right)=\left(a_{1} P_{12}\right) g_{2}-a_{2} \frac{M}{W_{2} \tau}
\end{aligned}
$$

The payoff function is consisted of two terms: the first term of $\pi_{i}$ denotes the gain of user $i$ with respect to the opponent's action, and the second term denotes the cost of with respect to its own action. Let $\pi\left(a_{1}, a_{2}\right)=\left(\pi_{1}\left(a_{1}, a_{2}\right), \pi_{2}\left(a_{1}, a_{2}\right)\right)$ be the payoff profile.

It is easy to check that, if this game will only be played for one time, the only Nash equilibrium (NE) is $(0,0)$, which means no one will respond to the other's request. According to the backward induction principle [9], this is also true when the repeated game will be played for finite times with game termination time known to both players. Therefore, in such scenarios, for each player, its only optimal strategy is to always play noncooperatively. However, in live streaming scenario, these two players will interact many rounds and no one can know exactly when its opponent will quit the game. Next, we show that, under a more realistic setting, besides the noncooperative strategy, cooperative strategies can be obtained. Let $s_{i}$ denote player $i$ s behavior strategy, and let $\mathbf{s}_{1}, \mathbf{s}_{2}$ denote the strategy profile. Next, we consider the following utility function of the infinitely repeated game:

$$
U_{i}(\mathbf{s})=\lim _{T \rightarrow \infty} \sum_{t=0}^{T} u_{i}(\mathbf{s})
$$

Now, we analyze NEs for the infinitely repeated game with utility function $U_{i}$. According to Folk theorem [9], for each feasible and enforceable payoff profile, there exists at least one Nash Equilibrium (NE) that can achieve it. The set of feasible payoff profiles for the above game is:

$$
\begin{gathered}
V_{0}=\text { convex hull }\left\{v \mid \exists\left(a_{1}, a_{2}\right) \text { with } \pi\left(a_{1}, a_{2}\right)=v\right. \\
\text { where } \left.a_{1}, a_{2} \in\{0,1\}\right\},
\end{gathered}
$$

and the set of enforceable payoff, denoted by $V_{1}$, is

$$
V_{1}=\left\{v \mid v \in V_{0} \quad \text { and } \quad v_{1}>0, v_{2}>0\right\} .
$$

Figure 1 illustrate the both feasible region and the enforceable region: the feasible region is inside the convex hull of $\left\{(0,0),\left(P_{21} g_{1},-\frac{M}{W_{2} \tau}\right),\left(P_{21} g_{1}-\frac{M}{W_{1} \tau}, P_{12} g_{2}-\frac{M}{W_{2} \tau}\right)\right.$, and $\left.\left(-\frac{M}{W_{1} \tau}, P_{12} g_{2}\right)\right\} . V_{1}$ is the gray region in Figure 1. It is clear that there exists an infinite number of Nash Equilibriums. For simplifications, in this paper, we use $\mathbf{x}=\left(x_{1}, x_{2}\right)$ to denote the set of NE strategies corresponding to the enforceable payoff profile $\left(x_{2} P_{21} g_{1}-x_{1} \frac{M}{W_{1} \tau}, x_{1} P_{12} g_{2}-x_{2} \frac{M}{W_{2} \tau}\right)$. 


\section{OPTIMAL STRATEGY ANALYSIS}

From the above analysis, we can see that the infinitely repeated game has an infinite number of Nash Equilibriums, and apparently, not all of them are simultaneously acceptable. For example, the payoff profile $(0,0)$ is not acceptable from both players' point of view. Therefore, in this section, we will discuss how to refine the equilibriums based on new optimality criteria to eliminate those less rational and likely Nash Equilibriums. Then, we will analyze the robustness of equilibriums against selfish users' cheating behavior and search for cheat-proof strategies.

\subsection{Nash Equilibrium Refinement}

In this paper, we consider the following optimality criteria: Pareto optimality, proportional fairness, and absolute fairness. Pareto Optimality: We first refine our solutions by applying the criterion of Pareto optimality. A payoff profile $v \in V_{0}$ is Pareto optimal if and only if there is no $v^{\prime} \in V_{0}$ that $v_{i}^{\prime} \geq v_{i}$ for all $i \in N$ [3]. Pareto Optimality means no one can increase his/her payoff without degrade others', which the rational players will always go to. For the game shown in Figure 1 , the segment between $\left(P_{21} g_{1},-\frac{M}{W_{2} \tau}\right)$ and $\left(P_{21} g_{1}-\right.$ $\left.\frac{M}{W_{1} \tau}, P_{12} g_{2}-\frac{M}{W_{2} \tau}\right)$ in the first quadrant and the segment between $\left(-\frac{M}{W_{1} \tau}, P_{12} g_{2}\right)$ and $\left(P_{21} g_{1}-\frac{M}{W_{1} \tau}, P_{12} g_{2}-\frac{M}{W_{2} \tau}\right)$ in the first quadrant are in the Pareto Optimal set.

Proportional Fairness: Next, we will further refine the solution set based on the criterion of proportional fairness. Here, a payoff profile is proportionally fair if the product $U_{1}(s) U_{2}(s)$ can be maximized, which can be achieved by maximizing $\pi_{1}(s) \pi_{2}(s)$ in every round. It has been shown in [3] that the proportional fairness solution is always Pareto optimal. The proportional fairness point $x^{*}$ can be derived by solving:

$$
\begin{aligned}
& \left.\frac{\partial \pi_{1}(s) \pi_{2}(s)}{\partial s_{1}}\right|_{x *}=0 \\
& =s_{2}\left(P_{12} P_{21} g_{1} g_{2}\right)-\frac{M^{2}}{W_{1} W_{2} \tau^{2}}-2 s_{1} P_{12} \frac{M}{W_{2} \tau}, \text { and } \\
& \left.\frac{\partial \pi_{1}(s) \pi_{2}(s)}{\partial s_{2}}\right|_{x *}=0 \\
& =s_{1}\left(P_{12} P_{21} g_{1} g_{2}\right)-\frac{M^{2}}{W_{1} W_{2} \tau^{2}}-2 s_{2} P_{21} \frac{M}{W_{1} \tau} .
\end{aligned}
$$

The solution is:

$$
\begin{aligned}
& x^{*}= \begin{cases}\left(\frac{C}{2}, 1\right) & \text { if } 1<\frac{2}{C}, \\
(1,1) & \text { if } \frac{2}{C} \leq 1 \leq \frac{C}{2}, \\
(1, c) & \text { if } 1 \geq \frac{C}{2},\end{cases} \\
& \text { where } C=\frac{M}{W_{2} \tau P_{12} g_{2}}+\frac{W_{1} \tau P_{21} g_{1}}{M} .
\end{aligned}
$$

Absolute Fairness: In many scenarios, absolute fairness is also an important criteria though an absolute fairness solution is not guaranteed to be Pareto optimal. In this paper, we consider absolute fairness in payoff, which refer to the most direct fairness criteria that all players in the game have the same payoff. By solving $U_{1}\left(x^{*}\right)=U_{2}\left(x^{*}\right)$, we can get the unique absolute fairness solution as follows:

$$
x^{*}=\left\{\begin{array}{lll}
\left(\frac{P_{21} g_{1} W_{2} \tau+M}{P_{12} g_{2} \tau W_{1}+M}, 1\right) & \text { if } & 1 \geq \frac{P_{21} g_{1} W_{2} \tau+M}{P_{12} g_{2} \tau W_{1}+M}, \\
\left(1, \frac{P_{12} g_{2} \tau W_{1}+M}{P_{21} g_{1} W_{2} \tau+M}\right) & \text { if } & 1 \geq \frac{P_{12} g_{2} \tau W_{1}+M}{P_{21} g_{1} W_{2} \tau+M} .
\end{array}\right.
$$

\subsection{Cheating and Cheat-Proof Strategies}

In Section 3.1, we obtained several unique equilibriums with different optimality criteria. However, as shown in (6) and (7), all these solutions involve some private information $\left(g_{i}, W_{i}\right)$ reported by each player. Due to players' greediness, honestly reporting private information cannot be taken for granted and players may tend to cheat whenever they believe cheating can increase their payoffs. Therefore, cheat proof is an essential requirement for $\mathrm{P} 2 \mathrm{P}$ live streaming social networks, and it is critical to design cheat-proof strategies that make cheating behavior nonprofitable and unattractive. Such a strategy can help stimulate user cooperation and improve the overall system performance.

Cheating On Private Information: One way of cheating is to cheat on the private information $\left(g_{i}, W_{i}, P_{j i}\right)$. First, let we exam whether the proportional fairness solution in (6) is cheat-proof with respect to $\left(g_{i}, W_{i}, P_{i j}\right)$. In (6), if $1<2 / C$, then $x_{2}^{*}=1$ is fixed. If $x_{1}^{*}=C / 2$, from the formula of $C$, it is clear that player 1 can reduce $x_{1}^{*}$ by reporting lower $g_{1}, W_{1}, P_{21}$ to increase his/her own payoff. Same situation also happens when $1>C / 2$, in which player 2 can cheat by reporting lower $g_{2}, P_{12}$, and $W_{2}$ to increase his/her own payoff. Applying the same examination to the absolute fairness solution in (7), we can also prove that the absolute fairness solution is also not cheat-proof with respect to the private information. Therefore, players have no incentives to honestly report their private information. On the contrary, they will cheat whenever cheating can increase their payoff.

As the consequence that both players cheat with respect to $W_{i}$ and $g_{i}$, from the above analysis, both players will report the minimum value of $g_{i}$ and $W_{i}$. Since we have assumed that $\operatorname{Pjig}_{i} \geq M /\left(W_{i} \tau\right)$, and $W_{i} \geq W_{\min }$, both players will claim $P_{j i} g_{i}=M / W_{i} \tau=M /\left(W_{\min } \tau\right)$ and both solution (7) and (6) becomes:

$$
x^{*}=(1,1)
$$

and the corresponding payoff profile is:

$$
v^{*}=\left(P_{21} g_{1}-\frac{M}{W_{1} \tau}, P_{12} g_{2}-\frac{M}{W_{2} \tau}\right),
$$

which implies that both players should always cooperate with each other. It is clear that solution in (8) forms an Nash Equilibrium, is Pareto-Optimal, and is cheat-proof with respect to private information $g_{i}$ and $W_{i}$.

Cheating On Buffer Information: The other way of cheating is to cheat on buffer information, that is, although player 
$i$ has the data in the buffer, he/she does not report it to its opponent, so that reduce the number of request from its opponent. As a result, increasing its own payoff by lowering $s_{i}$. This kind of cheating is effective only when at a certain round, both players request a chunk of data from each other. To prevent this kind of cheating, each player should not send data more than what the other one has sent.

Based on the above analysis, we can conclude that, in the two-player P2P live streaming game, in order to maximize each user's own payoff and be resistant to possible cheating behavior, a player should not send more data than what the other player has forwarded to him. Specifically, for each player in each round, it should always agree to send the requested data unless the other player refused it in the previous round or there is no useful data in the other player's buffer. We refer to the above strategy as two-player cheat-proof $P 2 P$ live streaming cooperation strategy.

\section{SIMULATION RESULTS}

In our simulation setting, there are total 500 users in the network without cooperation. Each peer is either an DSL peer with $768 \mathrm{kbps}$ uplink, or a cable peer with $300 \mathrm{kbps}$ uplink bandwidth. We fix the ration between DSL peers and cable peers as 4:6. The video is initially stored at an original server with upload bandwidth $3 \mathrm{Mbps}$. The request round is $1 \mathrm{sec}-$ ond and the buffer length is 30 seconds. We choose the "Foreman" video sequence $(352 \times 288)$ resolution with frame rate 30 frames per second. Using MPEG 4 to encode the video into a single layer bitstream with $150 \mathrm{kbps}$, and divided the video into 1-second chunks, thus the chunk size is $M=150 \mathrm{~KB}$. Among those peers, we randomly choose two peers to cooperate using the two-player cheat-proof P2P live streaming cooperation strategy. We set $g_{1}=1$, and every peer claim the lowest bandwidth $W_{\min }=300 \mathrm{kbps}$. In our simulations, we assume that when a selfish user lies about the chunks of data that are available in his/her buffer, he/she always lies about $m$ chunks of data in the buffer - even though these $m$ chunks of data are in the buffer, the selfish user tells the other player that he/she has not received them either.

Figure 4 shows the utilities of the cheating player when the other player is always honest. The $\mathrm{x}$ axis is the percentage of chunks in the buffer that the cheating player hides from the other one. The straight line is the utility if the player being honest, and the red triangle line is the utility if the player is cheating on the buffer information. It is clear that if the cheating player hides more data, it lowers his/her own payoff, and therefore, the best policy is being honest. This results shows the cheat-proof property of our proposed cooperation strategy. In addition, from the simulations, the average number of chunks that each user receives without cooperation is 20 , and with two-player cooperation, each user receives 36 chunks of data, which almost doubles the percentage of received data without cooperation. It shows that our strategy can improve each user's individual payoff and thus stimulate user cooper-

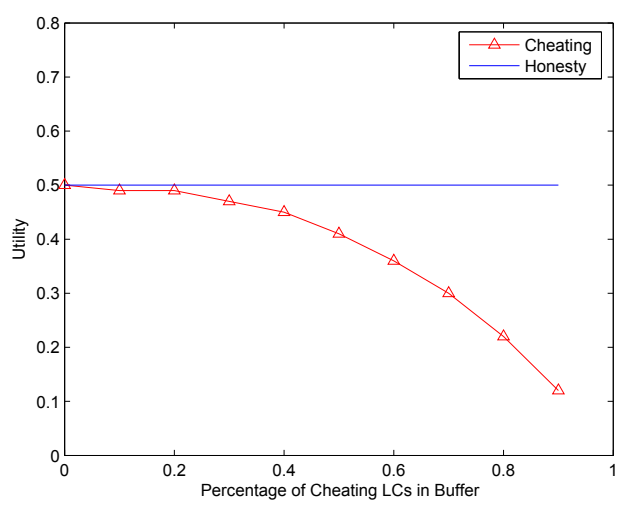

Fig. 2. Payoff of the cheating player and that of the honesty player. The $\mathrm{x}$ axis is the percentage of data chunks in the buffer that the cheating player hides from the other one.

ation.

\section{CONCLUSIONS}

In this paper, we investigate cooperation stimulation in $\mathrm{P} 2 \mathrm{P}$ live streaming social networks under a game theoretic framework. An illustrating two-player game is studied, and different optimality criteria, including Pareto-Optimality, proportional fairness and absolute fairness is performed to refine the obtained Nash Equilibriums. We also study the cheating behavior in P2P live streaming networks and propose a cheatproof strategy that makes noncooperation non-profitable, and thus unattractive, to selfish users. Our simulation results show that the proposed strategy is cheat proof, and all users improve their payoffs by cooperating with each other.

\section{REFERENCES}

[1] "The software is available at http://www.pplive.com/en/index.html," .

[2] G. Tan and S. A. Jarvis, "Apayment-based incentive and service differentiation mechanism for peer-to-peer streaming broadcast," In Proceedings of International Workshop on Quality of Service (IWQoS), June 2006.

[3] G. Owen, Game Theory, Academic Press, 3rd edition, 2007.

[4] Chiranjeeb Buragohain, Divyakant Agrawal, and Subhash Suri, "A game theoretic framework for incentives in $\mathrm{p} 2 \mathrm{p}$ systems," In Proceeding of the International Conference on Peer-to-Peer Computing, pp. 48-56, Sep 2003.

[5] S. Jun and M. Ahamad, "Incentives in bittorrent induce free riding," In Proceeding of the 2005 ACM SIGCOMM workshop on Economics of peer-to-peer systems, 2005.

[6] D. Qiu and R. Srikant, "Modeling and performance analysis of bittorrent-like peer-to-peer networks," In Proceedings of SIGCOMM 2004, 2004.

[7] Zhengye Liu, Yanming Shen, Shivendra Panwar, Keith Ross, and Yao Wang, "Using layered video to provide incentives in p2p live streaming," ACM Special Interest Group on Data Communication, August 2007.

[8] Ahsan Habib and John Chuang, "Incentive mechanism for peer-topeer media streaming," International Workshop on Quality of Service (IWQoS), pp. 171-180, June 2004.

[9] M.J. Osborne and A. Rubinste, A Course in Game Theory, The MIT Press, 1994. 